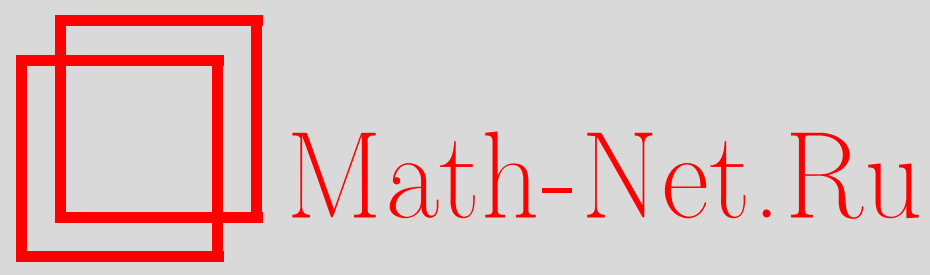

В. Н. Тютянов, О существовании разрешимых нормальных подгрупп в конечных группах, Матем. заметки, 1997, том 61, выпуск 5, 755-758

DOI: https://doi.org/10.4213/mzm1557

Использование Общероссийского математического портала Math-Net.Ru подразумевает, что вы прочитали и согласны с пользовательским соглашением http://www . mathnet.ru/rus/agreement

Параметры загрузки:

IP : 54.80 .73 .141

26 апреля 2023 г., 16:17:59 


\section{О СУЩЕСТВОВАНИИ РАЗРЕШИМЫХ НОРМАЛЬНЫХ ПОДГРУПП В КОНЕЧНЫХ ГРУППАХ}

\section{В.Н. Тютянов}

Рассматривается строение конечной группы в зависимости от строения подгрупп, порожденных элементами классов сопряженности.

Библиография: 5 названий.

Строение конечной групш существенно зависит от строения подгрупп, порожденных ее элементами класса сопряженности. Примером этого является хорошо известная теорема Бэра [1, теорема 2.66]. Изучая конечные группы порожденные сопряженньп классом 3 -транспозиций, Б. Фишер $[1,2.8]$ открыл три новые спорадические групшы. Настоящая работа примыкает к данной тематике. В ней получены результаты, дополняюшие теорему Бэра.

ТЕорема 1. Пусть $G$ - конечная р-разреиимая группа для некоторого $p \in \pi(G)$. Если $\left\langle x, x^{g}\right\rangle$ - группа нечетного порядка для всех $g \in G$ и некоторого $р$-әлемента $x \in G$, mo $x \in O(G)$.

Теорема 2. Пусть $G$-конечная группа, силовские р-подгруппы которой абелевы для некоторого $p \in \pi(G)$. Пусть $1 \neq x \in G, 1 \neq y \in G$. Eсли $\left\langle x^{g}, y^{h}\right\rangle-p$-группа для всех $g, h \in G u\left\langle x^{G}\right\rangle=\left\langle y^{G}\right\rangle$, mo $\langle x, y\rangle \subseteq O_{p}(G)$.

Все рассматриваемые группы конечны. Обозначения, в основном, стандартны. Если $x_{1}, \ldots, x_{n}$ - элементы группы $G$, то $\left\langle x_{1}, \ldots, x_{n}\right\rangle$ - группа порожденная этими элементами; $\left\langle x^{G}\right\rangle=\left\langle x^{g} \mid g \in G\right\rangle$ - нормальное замыкание элемента $x$ в групше $G ; \pi(G)-$ множество всех простых делителей порядка грушшы $G$.

1. Доказательство теоремы 1. Пусть $G$-минимальный контрпример к теореме 1 . Без ограничения общности можно считать, что $|x|=p$ - простое число.

(1) $O(G)=1$.

ДокАЗАтЕльСтво. Пусть $O(G) \neq 1$. Обозначим $\bar{G}=G / O(G)$. Так как $x \notin O(G)$, то $x O(G)=\bar{x} \neq 1$. Поскольку $G$ является минимальным контрпримером к теореме 1 , то $\bar{x} \in O(\bar{G})$, а значит, $x \in O(G)$. Противоречие.

(2) $O_{2}(G)=1$. 
ДоказАтельство. Пусть $O_{2}(G) \neq 1$. Рассмотрим в группе $G$ подгрупшу $O_{2}(G)\langle x\rangle$. Если $G=O_{2}(G)\langle x\rangle$, то так как $\left\langle x, x^{g}\right\rangle$ - группа нечетного порядка для любого $g \in G$, то $\left\langle x, x^{g}\right\rangle=\langle x\rangle\left(\left\langle x, x^{g}\right\rangle \cap O_{2}(G)\right)=\langle x\rangle$. Теперь ясно, что $\langle x\rangle \unlhd G$ и $x \in O(G)$. Противоречие.

Значит, $O_{2}(G)\langle x\rangle \neq G$ и $x \in O\left(O_{2}(G)\langle x\rangle\right)$. Поэтому $O_{2}(G)\langle x\rangle=O_{2}(G) \times\langle x\rangle$, а следовательно, $x \in C_{G}\left(O_{2}(G)\right) \unlhd G$.

(a) $C_{G}\left(O_{2}(G)\right) \neq G$.

В этом случае $x \in O\left(C_{G}\left(O_{2}(G)\right)\right)$. Поскольку $O\left(C_{G}\left(O_{2}(G)\right)\right) \subseteq O(G)$, то $x \in O(G)$. Последнее невозможно.

(b) $C_{G}\left(O_{2}(G)\right)=G$.

Рассмотрим фактор-группу $\bar{G}=G\left(O_{2}(G)\right)$. Тогда $\bar{x} \in O(\bar{G})$. Теперь рассмотрим полньй прообраз $L$ грушшы $O(\bar{G})$ в $G$. Поскольку $O(L) \subseteq O(G)$, то согласно (1) $O(L)=1$. Однако, $O(L) \neq 1$. Противоречие.

(3) Группа $G=N \lambda\langle x\rangle$, где $N$ - простая неабелева группа.

ДокАЗАТЕЛЬСтво. Поскольку $G$ - $p$-разрешимая группа, то $G$ не является простой неабелевой группой. Пусть $N$ - минимальная нормальная подгруппа в $G$. Как следует из (1) и (2), $N=N_{1} \times \cdots \times N_{f}$, где $N_{i}$ - изоморфные простые неабелевы групшы. Поскольку $G-p$-разрешимая группа, то порядки ее неабелевых факторов не делятся на $p$. Отсюда $x \notin N$. Рассмотрим грушп $N\langle x\rangle$.

(a) $N\langle x\rangle \neq G$.

В этом случае $x \in O(N\langle x\rangle)$ и $O(N\langle x\rangle)=\langle x\rangle(N \cap O(N\langle x\rangle))$. Но $N \cap O(N\langle x\rangle)=1$, поэтому $O(N\langle x\rangle)=\langle x\rangle$. Теперь видно, что $x \in C_{G}(N)$. Если $C_{G}(N) \neq G$, то $x \in O\left(C_{G}(N)\right)$, а значит, $x \in O\left(C_{G}(N)\right) \subseteq O(G)=1$. Противоречие. Таким образом, $C_{G}(N)=G$. Так как $N$-неабелева группа, то это невозможно.

(b) $N\langle x\rangle=G$.

В этом случае $G=N \lambda\langle x\rangle$. Групша $\langle x\rangle$ действует транзитивно на множестве $\left\{N_{1}\right.$, $\left.\ldots, N_{f}\right\}$. Значит, $f=p$, или $f=1$. Пусть сначала $f=p$.

Поскольку группа $\langle x\rangle$ действует транзитивно на $\left\{N_{1}, \ldots, N_{p}\right\}$, то при подходящей нумерации получим $N_{i+1}=N_{1}^{x^{i}}$, где $i=0,1, \ldots, p-1$. Значит, $N=N_{1} \times N_{1}^{x} \times \cdots \times N_{1}^{x^{p-1}}$. Пусть теперь $t_{1}$ инволюция из $N_{1}$. Групша $\left\langle x, x^{t_{1}}\right\rangle$ - нечетного порядка. Рассмотрим элемент $x^{-1} x^{t_{1}} \in\left\langle x, x^{t_{1}}\right\rangle$. Очевидно, $1 \neq\left(x^{-1} t_{1} x\right) t_{1} \in N_{1}^{x} \times N_{1}$ является инволюцией, что невозможно, так как порядок $\left\langle x, x^{t_{1}}\right\rangle$ - нечетное число. Значит, $f=1$, что доказьвает (3).

(4) Минимального контрпримера к теореме 1 не существует.

ДоказАтельство. Согласно (3) $G=N \lambda\langle x\rangle$, где $N$ - простая неабелева группа и $(|N|,|x|)=1$. Поэтому $\langle x\rangle$ нормализует некоторую силовскую 2 -подгрупу $S$ из $N$. Поскольку $G$ - минимальный контрпример к теореме 1 , то $\langle x\rangle$ централизует подгруппу $S$. Согласно результату С. А. Сыскина (см. [2, теорема 2]) группа $N$ изоморфна группе лиева типа нечетной характеристики. Так как порядок группы диагональных автоморфизмов грушш лиева типа всегда делит ее порядок (см. таблицу в [3, с. 46]), то $x \notin \operatorname{Inn} \operatorname{diag}(N)$. Согласно [4, лемма 7.3$]$ возможна одна из следуюших ситуаций.

1) $|x|=3, N \cong D_{4}\left(3^{n}\right)$ и $x$ либо автоморфизм графа, либо графо-полевой автоморфизм. 
Этот случай невозможен, поскольку $(|x|,|N|)=1$.

2) $|x|=3, N \cong{ }^{3} D_{4}\left(3^{n}\right)$ и $x$ - автоморфизм графа.

Этот случай невозможен, поскольку $(|x|,|N|)=1$.

3) $x$ - полевой автоморфизм.

Пусть $\mathrm{GF}\left(q^{m}\right)$ - конечное поле характеристики $q$, над которьм определена группа лиева типа $N$. В этом случае найдется $\gamma \in \operatorname{Aut}\left(\operatorname{GF}\left(q^{m}\right)\right)$ такой, что отображение корневых подгрупп $X_{\varepsilon}(t) \rightarrow X_{\varepsilon}\left(t^{\gamma}\right)$ (для всех $\varepsilon \in \Sigma$ - система положительньг корней) продолжается до полевого автоморфизма $x$ группы $N$. Поэтому $x$ нормализует каждую корневую подгруппу $X_{ \pm \varepsilon}$, где $\varepsilon \in \Sigma$.

Если $N \supsetneqq \mathrm{PSL}_{2}\left(q^{m}\right)$, то так как $G$ - минимальный контрпример к теореме $1, x$ будет централизовать групу $\left\langle X_{\varepsilon}, X_{-\varepsilon}\right\rangle$ для всех $\varepsilon \in \Sigma$. Таким образом, $\left[x, X_{ \pm \varepsilon}\right]=1$ для всех $\varepsilon \in \Sigma$. Отсюда следует, что $[x, N]=1$. Последнее невозможно. Значит, $N \cong \mathrm{PSL}_{2}\left(q^{m}\right)$, где $m=a p$. Пусть $\tau$ - инволющия, не коммутируюшая с $x$. Тогда $\tau \tau^{x} \in\langle x, \tau\rangle=\left\langle x, x^{\tau}\right\rangle \lambda\langle\tau\rangle=(L \lambda\langle x\rangle) \lambda\langle\tau\rangle=(L \lambda\langle\tau\rangle) \lambda\langle x\rangle$, где $N \supset L$-x-допустимая группа. Согласно теореме Диксона [5, теорема 8.27] $L$ - абелева группа нечетного порядка. Поэтому $\tau \tau^{x}=u \in L$. Так как $L$ - абелева, допустимая относительно $x$ груша, то $u u^{x}=u^{x} u$. Отсюда $\tau \tau^{x} \tau^{x} \tau^{x^{2}}=\tau^{x} \tau^{x^{2}} \tau \tau^{x}$. Следовательно, $\tau^{x} \tau \tau^{x^{2}}=\tau^{x^{2}} \tau \tau^{x}$.

Рассмотрим матрицу

$$
T=\left[\begin{array}{cc}
\alpha & 1 \\
-1-\alpha^{2} & -\alpha
\end{array}\right]
$$

где $\alpha$ - произвольный элемент поля $\mathrm{GF}\left(q^{m}\right)$, и пусть инволюция $\tau$ ее образ в $\mathrm{PSL}_{2}\left(q^{m}\right)$. Условие $\tau^{x} \tau \tau^{x^{2}}=\tau^{x^{2}} \tau \tau^{x}$ влечет $T^{x} T T^{x^{2}}= \pm T^{x^{2}} T T^{x}$, где

$$
T^{x}=\left[\begin{array}{cc}
\bar{\alpha} & 1 \\
-1-\bar{\alpha}^{2} & -\bar{\alpha}
\end{array}\right], \quad T^{x^{2}}=\left[\begin{array}{cc}
\overline{\bar{\alpha}} & 1 \\
-1-\overline{\bar{\alpha}}^{2} & -\overline{\bar{\alpha}}
\end{array}\right] .
$$

Черта означает действие автоморфизма Фробениуса на элемент поля $\operatorname{GF}\left(q^{m}\right)$. Матрица $T^{x^{2}}$ получается из матрицы $T^{x}$ заменой $\bar{\alpha}$ на $\overline{\bar{\alpha}}$. Поэтому матришы $C=T^{x} T T^{x^{2}}$ и $T^{x^{2}} T T^{x}$ получаются друг из друга перестановкой $\bar{\alpha}$ и $\overline{\bar{\alpha}}$.

Пусть сначала $T T^{x} T^{x^{2}}=T^{x^{2}} T T^{x}$. Тогда имеет место равенство

$$
C_{22}=-\alpha-\alpha \bar{\alpha}^{2}+\bar{\alpha}+\alpha^{2} \bar{\alpha}+\overline{\bar{\alpha}}+\bar{\alpha}^{2} \overline{\bar{\alpha}}-\alpha \bar{\alpha} \overline{\bar{\alpha}} .
$$

Отсюда получаем

$$
-\alpha-\alpha \bar{\alpha}^{2}+\bar{\alpha}+\alpha^{2} \bar{\alpha}+\overline{\bar{\alpha}}+\bar{\alpha}^{2} \overline{\bar{\alpha}}-\alpha \bar{\alpha} \overline{\bar{\alpha}}=-\alpha-\alpha \overline{\bar{\alpha}}^{2}+\overline{\bar{\alpha}}+\alpha^{2} \overline{\bar{\alpha}}+\bar{\alpha}+\overline{\bar{\alpha}}^{2} \bar{\alpha}-\alpha \bar{\alpha} \overline{\bar{\alpha}},
$$

или

$$
\alpha^{2} \bar{\alpha}-\alpha \bar{\alpha}^{2}+\bar{\alpha}^{2} \overline{\bar{\alpha}}=-\alpha \overline{\bar{\alpha}}^{2}+\alpha^{2} \overline{\bar{\alpha}}+\bar{\alpha} \overline{\bar{\alpha}}^{2} .
$$

Положив $\bar{\alpha}=\alpha^{q^{a}}$, получим $\overline{\bar{\alpha}}=\alpha^{q^{2 a}}$. Подставив значения $\bar{\alpha}$ и $\overline{\bar{\alpha}}$ в равенство $(*)$, имеем

$$
\alpha^{2 q^{2 a}+q^{a}}-\alpha^{2 q^{2 a}+1}-\alpha^{q^{2 a}+2 q^{a}}+\alpha^{q^{2 a}+2}+\alpha^{2 q^{a}+1}-\alpha^{q^{a}+2}=0 .
$$

Уравнение

$$
z^{2 q^{2 a}+q^{a}}-z^{2 q^{2 a}+1}-z^{q^{2 a}+2 q^{a}}+z^{q^{2 a}+2}+z^{2 q^{a}+1}-z^{q^{a}+2}=0
$$


степени $2 q^{2 a}+q^{a}$ имеет не более $2 q^{2 a}+q^{a}$ корней в поле $\operatorname{GF}\left(q^{a p}\right)$.

Случай $T^{x} T T^{x^{2}}=-T^{x^{2}} T T^{x}$ также приводит к полиномиальному уравнению для $\alpha$ степени $2 q^{2 a}+q^{a}$. Поскольку всякий элемент поля $\operatorname{GF}\left(q^{a p}\right)$ должен удовлетворять одному из этих полиномиальных уравнений, то должно вьполняться неравенство $2\left(2 q^{2 a}+q^{a}\right) \geqslant q^{a p}$. Так как $p$ и $q$ различные нечетные простые числа, то легко видеть, что данное неравенство не выполняется ни при каких значениях параметра a. Противоречие. Таким образом, минимального контрпримера к теореме 1 не существует.

Теорема 3. Пусть $G$ - конечная разрешимая группа. Если $\left\langle x, x^{g}\right\rangle-$ группа нечетного порядка для всех $g \in G$, то $x \in O(G)$.

ДокАЗАТЕЛЬСТво. Пусть $G$ - минимальный контрпример к теореме 3 . Дословное повторение пунктов (1) и (2) теоремы 1 приводит к тому, что $O(G)=O_{2}(G)=1$. Последнее невозможно в разрешимой групше.

2. Доказательство теоремы 2. Поскольку $\left\langle x^{G}\right\rangle=\left\langle y^{G}\right\rangle \unlhd G$, а также $\left[x, y^{g}\right]=1$ для всех $g \in G$, то $1 \neq x \in Z\left(\left\langle y^{G}\right\rangle\right) \operatorname{char}\left\langle y^{G}\right\rangle \unlhd G$. Отсюда следует, что $x \in O_{p}(G)$. Аналогично доказьвается, что $y \in O_{p}(G)$. Таким образом, $\langle x, y\rangle \subseteq O_{p}(G)$.

ЗАмЕчАнИЕ. Как показывает пример групшы $S_{6}$, условие абелевости $p$-силовских подгрупп в общем случае нельзя опустить. Действительно, если положить $x=(1,2)$; $y=(1,2)(3,4)(5,6)$, то $\left\langle x^{g}, y^{h}\right\rangle-2$-группа для всех $g, h \in S_{6}$. Однако, $\langle x, y\rangle \not \subset O_{2}\left(S_{6}\right)$.

Гомельское отделение ВЦ АН Беларуси

Поступило 01.03 .95

Исправленный вариант 20.03.96

\section{СПИСОК ЦИТИРОВАННОЙ ЛИТЕРАТУРЫ}

[1] Горенстейн Д. Конечные простые группы. Введение в их классификацию. М.: Мир, 1985.

[2] Сыскин С. А. Об автоморфизмах конечных групп // Сиб. матем. ж. 1991. Т. 32. №6. C. 164-168.

[3] Картер Р. Простые группы и простые алгебры Ли // Математика. 1966. Т. 10. № 5. С. 3-47.

[4] Gorenstein D., Lyons R. The local structure of finite groups of characteristic 2 type. Mem. Amer. Math. Soc. V. 276, 1983.

[5] Huppert B. Endliche Gruppen. I. Berlin-New York: Springer Verlag, 1967. 